\title{
BMJ Open Vietnamese medical students and binge drinking: a qualitative study of perceptions, attitudes, beliefs and experience
}

\author{
Thi Thu Huong Nguyen, ${ }^{1}$ Marguerite C Sendall, ${ }^{2}$ Katherine M. White, ${ }^{1}$ \\ Ross McD Young ${ }^{3}$
}

To cite: Nguyen TTH, Sendall MC, White KM., et al. Vietnamese medical students and binge drinking: a qualitative study of perceptions, attitudes, beliefs and experience. BMJ Open 2018;8:e020176. doi:10.1136/ bmjopen-2017-020176

- Prepublication history and additional material for this paper are available online. To view please visit the journal (http:// dx.doi.org/10.1136/bmjopen2017-020176).

Received 18 0ctober 2017 Revised 21 March 2018 Accepted 6 April 2018

\section{Check for updates}

${ }^{1}$ School of Psychology and Counselling, Faculty of Health, Institute of Health and Biomedical Innovation, Queensland University of Technology, Brisbane, Queensland, Australia ${ }^{2}$ School of Public Health and Social Work, Faculty of Health, Institute of Health and Biomedical Innovation, Queensland University of Technology, Brisbane, Queensland, Australia ${ }^{3}$ Faculty of Health, Institute of Health and Biomedical Innovation, Queensland University of Technology, Brisbane, Queensland, Australia

Correspondence to Thi Thu Huong Nguyen; tt26.nguyen@hdr.qut.edu.au

\section{ABSTRACT}

Objectives To explore the perceptions, attitudes, beliefs, and experiences related to Vietnamese medical students' binge drinking.

Design A qualitative study comprising semi-structured focus groups/interviews with medical students and semistructured interviews with key informants. Thematic analysis of data.

Setting Participants were a convenience sample of usual volunteers from a medical university in Viet Nam.

Participants 19 medical students from year 1 to 6 and 4 key informants agreed to participate in the study. Results The study found participants believe medical students drink less than other students and are not binge drinkers yet they experience and/or witness many binge drinking occasions among medical students. Participants consider alcohol use as culturally acceptable in Vietnamese society and a way for medical students to create and improve relationships with their friends, teachers, or work colleagues. Group affiliation and peer pressure to drink excessive alcohol are identified among medical students, especially male students.

Conclusion The culture of drinking behaviour was explored among medical students in Viet Nam. This study reveals a dichotomy between the belief of not being binge drinkers and the experience of many binge drinking occasions among medical students. This tension suggests future research about binge drinking behaviour of Vietnamese medical students is required.

\section{INTRODUCTION}

Harmful alcohol consumption such as binge drinking has been recognised as a significant risky behaviour among adolescents and young adults, increasing the likelihood of health problems, drink driving, unsafe sexual behaviour, and accidents. ${ }^{1}$ Risky drinking during adolescence and young adulthood predicts future alcohol-related problems and academic failure in certain groups. ${ }^{2}$

Binge drinking is highly prevalent among college and university students, ${ }^{34}$ including
Strengths and limitations of this study

- This study was the first qualitative study to explore factors underlying binge drinking behaviour among Vietnamese medical students drawing from the Theory of Planned Behaviour.

- As this study comprised a convenience sample of usual volunteers recruited from only one medical university, the results may not be representative of all medical students in Viet Nam

- Selection bias may have occurred due to the purposive selection of medical students for the focus groups by the medical school administrative staff.

- The gender of the interviewer (female) may have affected the stories shared by participants and the interpretation of the data.

medical students. ${ }^{5} 6$ Medical students consider alcohol use as a means of coping to mark the transition from adolescence to adulthood, ${ }^{7}$ and to cope with the stress and strain of medical education. ${ }^{8}$ Risky drinking behaviours among medical students is concerning because alcohol consumption increases from medical school into their early professional careers. ${ }^{9}$ Drinking behaviours among medical students have implications for the health of the general population. Medical students' own drinking behaviours might shape their beliefs about normal or safe consumption. Medical practitioners' attitudes, beliefs and experience of alcohol, and their own drinking behaviour may influence patient counselling practices related to alcohol. ${ }^{10}$ Physicians and medical students with a healthy lifestyle were more likely to have a positive attitude towards preventive counselling and were more confident in practicing preventive counselling, including related to alcohol. ${ }^{11-13}$ Medical students who drank excessively were less likely to discuss alcohol 
with their patients or to think it relevant to do so. ${ }^{14}$ However, the researchers noted that routine training in relation to alcohol misuse screening and counselling for medical students may help improve their future practice and beliefs for alcohol counselling. ${ }^{14}$ Understanding drinking behaviours and factors underlying these behaviours is needed for this population.

Numerous international studies have reported data about drinking behaviour in medical students for many countries, but there is scant data about drinking behaviour and determinants of this behaviour in the Vietnamese medical student population. A study reported $18.4 \%$ of medical students in Hai Phong province were classified as experiencing alcohol misuse. ${ }^{15}$ Another study found $12.5 \%$ of medical students in Hanoi and Thai Nguyen provinces reported alcohol problems. ${ }^{16}$ The literature about alcohol consumption in Viet Nam contains little analysis of the factors which influence alcohol use among young adults, especially university students. A recent study among Vietnamese students (including medical students) indicates drinking in large groups and with casual acquaintances were associated with a significantly higher level of alcohol consumption. ${ }^{17}$ Coping, social, and enhancement motives were associated with a higher level of alcohol consumption among both genders. ${ }^{18}$ Peer pressure is reported as a frequent reason for drinking among Vietnamese people and can influence how much young people drink. ${ }^{19}$

Research has identified many health behaviour models to explain and predict alcohol use, and provide a framework for successful drinking behaviour change strategies. The Theory of Planned Behaviour (TPB) (Ajzen, 1985, $1991)^{2021}$ is one such model. The TPB posits behaviour as a function of intention determined by three factors: attitude (regarding whether the target behaviour will result in certain desirable outcomes), subjective norm (perception that salient social agents engage the individual in the target behaviour), and perceived behavioural control (PBC) (perceptions about whether the individual has the resources to engage in the target behaviour). Each factor influencing intention is supported by salient underlying beliefs including behavioural beliefs (attitude; advantages and disadvantages of performing the target behaviour), normative beliefs (subjective norm; key referents who approve or disapprove of performing the target behaviour), and control beliefs (PBC; barriers or facilitators to perform the target behaviour). The TPB has demonstrated its effectiveness in predicting drinking intentions and behaviour among student populations, ${ }^{22} 23$ and informed the design of many interventions in relation to drinking behaviour. ${ }^{24-27}$

To the authors' knowledge, there have been no studies published which examine factors underlying drinking behaviour among Vietnamese medical students using an established health behaviour model. Most studies have used self-report surveys without rich accounts of students' drinking perceptions and experiences. This study applies a qualitative methodology to explore the perceptions, attitudes, and experiences in relation to Vietnamese medical students' binge drinkingi, ${ }^{, 2}$ and identify salient beliefs about binge drinking among Vietnamese medical students informed by the Theory of Planned Behaviour. The results from this study will assist our understanding of the culture of drinking behaviour among Vietnamese medical students for intervention development.

\section{METHODS}

This qualitative study was conducted at a medical university in Viet Nam during October to November 2016.

\section{Participants}

On behalf of the first author, a staff member of the University Centre for Educational Testing and Quality Assurance contacted approximately 30 eligible students previously willing to assist with research and other university activities by email or in person to inform them about the study. The students were provided with a recruitment flyer and a consent form to complete with contact details. If only one student was available for a focus group or the student preferred an interview, an interview was conducted. The students were asked to give the form to the staff member to pass onto the first author. The first author contacted students who agreed to participate in the study by phone and arranged a suitable time to conduct the focus groups/ interviews.

The first author visited five key informants working at the university (the Manager of a relevant health research Institute, the Manager of Healthcare Centre, the Deputy Head of the Dormitory and Student Life Management Office, a Lecturer, and the Leader of Student Association) to inform them about the study, and invite them to participate. The key informants were invited to participate in the research because their work is associated with medical students and their likely understanding about medical students' lives and behaviours. If the key informants agreed to participate in the study, the first author arranged a time and place to conduct the interviews.

\section{Patient and public involvement}

No patients or public were involved in my study.

\section{Data collection}

Focus groups/interviews with medical students were conducted in separate groups of males and females at a meeting room with soundproofing at the University Centre of Educational Testing and Quality Assurance. Each focus group consisted of 4 to 6 participants. Each focus group/interview lasted 45-60 min. Before conducting focus groups/interviews, medical students were asked to complete a brief written survey of TPB belief

\footnotetext{
${ }^{\mathrm{i}}$ Binge drinking in this study is defined as consuming more than five standards drinking (SDs) per occasion and a SD contains 10 grams of alcohol (12.5 mL of pure alcohol) equal to $330 \mathrm{~mL}$ beer $(5 \%)$ or one glass $(100 \mathrm{~mL})$ of wine $(13.5 \%)$ or one shot $(40 \mathrm{~mL})$ of spirit $(40 \%-43 \%) .^{28}$
} 
elicitation questions (see Ajzen, 2006) ${ }^{29}$ and the answers were discussed in depth in the focus groups or interviews. For example, some medical students mentioned in the survey that peer pressure was a facilitator of their binge drinking. Then, in the focus groups/interviews, the researcher asked participants to describe more about the drinking occasions where they experienced peer pressure and their feelings about this situation. At the end of the focus groups or interviews, participants were asked to share any additional information in relation to the salient TPB belief elicitation questions. A semi-structured interview guide including questions about the perceptions, attitudes, and experiences of medical students towards binge drinking behaviour among medical students was used (online supplementary appendix 1). To create a comfortable environment, the first author gave the participants $15 \mathrm{~min}$ prior to the focus group/interview to have a light snack, chat about their lives and study, and fill in the brief written survey. Interviews with key informants were conducted at their private offices. Each interview lasted 30-45 min.

The focus groups and interviews were conducted in Vietnamese by the first author who is a young female and has experience in conducting qualitative research. All focus groups and interviews were conducted in Vietnamese and recorded. These focus groups and interviews were conducted until similar themes emerged indicating saturation had been achieved. This study comprised four focus groups with 17 medical students. Two medical students preferred to be interviewed. A description of age, gender, and past binge drinking behaviour of medical students participating in this study is provided in online supplementary appendix 2.

Four key informants agreed to participate in the interviews: The Manager of a relevant health research Institute, the Deputy Head of the Dormitory and Student Life Management Office, a Lecturer, and the Leader of Student Association. The Manager of the Health Centre declined to participate in the interview because the Manager had no experience of students with alcohol problems.

\section{Data analysis}

The focus groups and interviews were transcribed verbatim and the transcriptions were translated into English by the first author. Thematic analysisi ${ }^{\mathrm{ii}}$ was undertaken in several steps to structure and categorise contents. ${ }^{30}$ The coding and grouping process was conducted by another researcher with experience in qualitative analysis to ensure

\footnotetext{
${ }^{\mathrm{ii}}$ For thematic analysis, the first author read the transcriptions and field notes several times to understand it as a whole and identify essential codes. Reading and re-reading the text line-by-line and open coding were conducted. Meaningful codes referring to the same idea were identified and sorted into groups relevant to the aim of the study and the questions of the focus groups and interviews. Axial coding was conducted by grouping similar codes into sub-categories and later merged into main categories. Finally, all categories were unified around a major category, which represented the main theme of the study.
}

soundness and trustworthiness. The inter-rater reliability reached $89 \%$. The first author discussed the results with the other authors to finalise emerging themes. Emergent themes were analysed to identify recurring themes, their relationships to each other, and clear and hidden meanings. Each emergent theme was labelled to express the essential similarities and conceptual encapsulation.

The Results section provides quotations for illustrative purposes. Each focus group/interview with medical students and interview with key informants (and related quotations) is identified by a unique code. Codes were constructed using the abbreviation of a focus group (FG) or interview (IV) and the order of the focus group/interview, and the abbreviation of gender (M-male and F-female). For example, FG03-M indicates a male student (M) participated in the third focus group (FG03). IV01 and IV02 were the interviews of medical students. IV03 to IV06 were the interviews of key informants.

\section{RESULTS}

This study identified the salient TPB beliefs obtained from the brief written survey (see online supplementary appendix 3).

The data analysis revealed four themes which shed light on medical students and key informants' perceptions, attitudes, experiences, and beliefs about binge drinking behaviour among Vietnamese medical students. Interestingly, medical students and key informants who participated in this study reported the same opinions in relation to binge drinking behaviour among Vietnamese medical students. Therefore, in this section, the word 'participants' is used to refer to both medical students and the key informants. Themes 1, 2 and 3 help understand participants' perceptions, attitudes, and experience about binge drinking behaviour. The salient TPB beliefs underlying binge drinking among these medical students are identified in all themes (figure 1).

\section{Medical students drink less than other students: 'We are not binge drinkers'}

Most participants believe medical students drink less than students at other universities and binge drinking happens infrequently. In their view, "students in the universities which relate to technology or have more male students than females often drink much more alcohol...' (FG02-F). Students from other universities which have more groups/teams/clubs and more activities than medical students would have more opportunities to meet each other and have parties' (FG03-M) in which alcohol is often consumed. Participants mention medical students are not binge drinkers because they only drink alcohol on a few occasions during a year and there are few activities or events at the university where students gather and drink to celebrate.

Participants think medical students do not drink much alcohol because they 'all are good and study hard at the campus and for their practice in hospitals' (IV05-F) and 'have less free time to go out and have a drink' (FG03-M). The participants 


\section{Objectives}

\section{Themes}

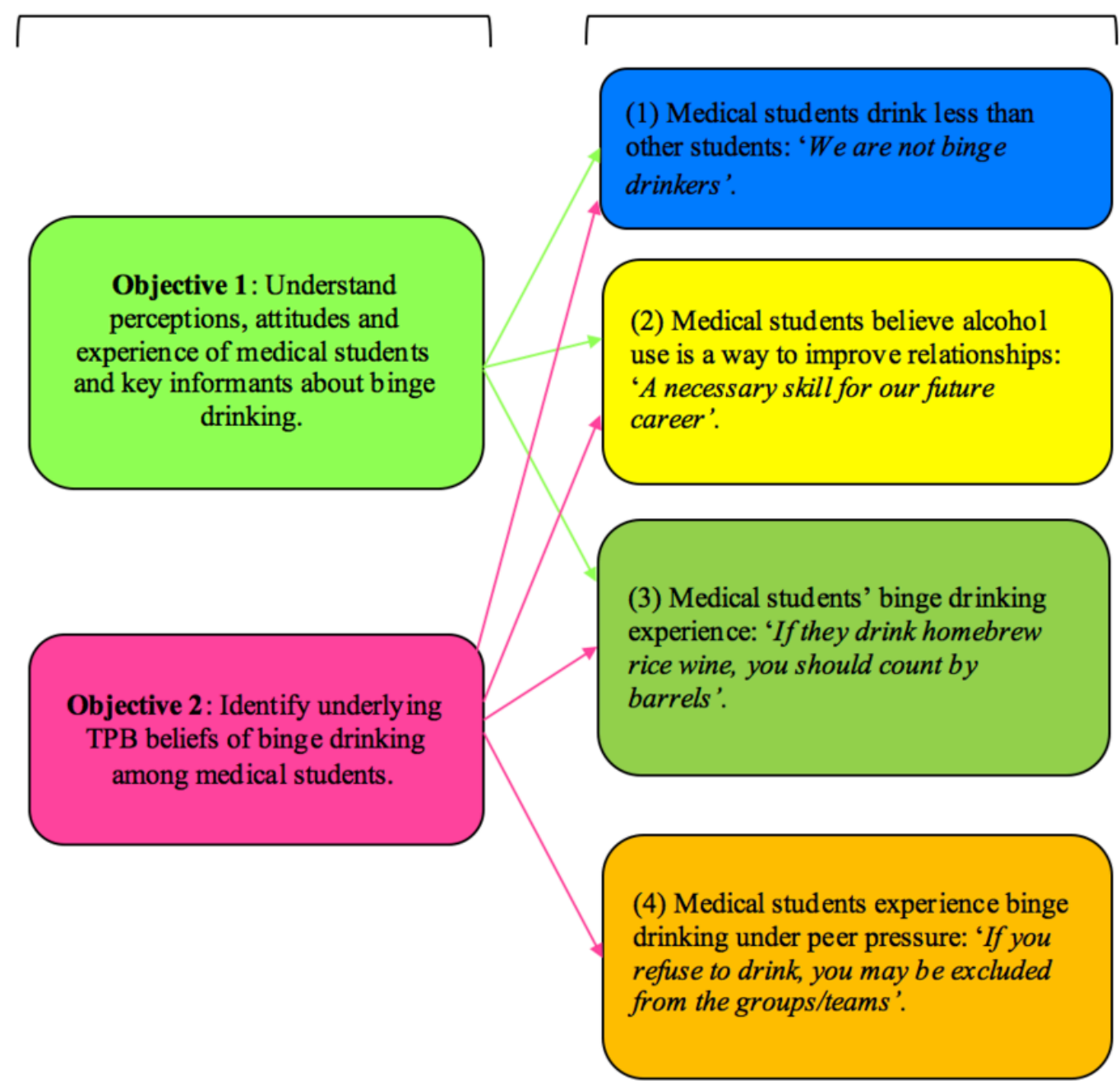

Figure 1 The study's themes and objectives.

believe medical students understand the disadvantages of alcohol to their body through their studies at the medical university. They suppose " medical students have higher knowledge about this issue and they may take care about their health more than others' (FG04-F); and this helps control their decisions in relation to binge drinking. Participants feel confident with their knowledge about alcohol, but most do not know the definition of binge drinking or think consuming more than five drinks per occasion is considered binge drinking behaviour.

The participants perceive drinking behaviour can influence medical students' image and career in the future. It is the reason why they assume medical students rarely join in binge drinking occasions.

FG03-M 'Medical students don't want to get a fine relating to drinking because they are afraid of its influence on their image'

Some female participants believe drinking too much alcohol can affect their image as an oriental woman. Vietnamese traditional culture recognises females as four virtues of 'Industriousness - Appropriate self-presentation
- Communication skills - Behaviour' ('Công - Dung Ngôn - Hạnh'). It is not culturally acceptable for Vietnamese women to drink alcohol and get drunk because this behaviour can lead to 'many bad behaviours and unwanted results' (FG02-F). This is one reason female participants do not consume much alcohol at drinking occasions.

In summary, participants believe medical students drink less alcohol than other students and do not binge drink frequently because they have less free time and are concerned more about the impact of drinking behaviour on their health, study, lives and image as future doctors. In particular, female medical students consider the effect of alcohol consumption on their image as Vietnamese ideal oriental women. The concerns the medical students raise serve as examples of TPB control beliefs which prevent them from binge drinking.

Medical students believe alcohol use is a way to improve relationships: 'A necessary skill for our future careers'.

The participants believe alcohol use among medical students is a 'normal behaviour' or 'a social tradition'(IV05-F) 
as it is an accepted cultural aspect of Vietnamese people. Drinking alcohol is considered as 'a way medical students use to talk with each other' (IV03-M) and helps people to speak freely: 'A shot of drink starts the ball rolling' (FG01M). Participants believe alcohol consumption is a way to create or improve their friendships and other relationships, and a necessary skill for their future career because becoming a strong drinker may help them be appreciated by their future boss and colleagues. In drinking occasions, they drink more alcohol to show drinking ability or respect to the people they are drinking with, in particular older people. The beliefs raised by the participants are consistent with the TPB control beliefs which enable medical students to binge drink.

FG04-F"In the future, we have to work and communicate with others and we generally need a drink to respect our partners"

A male participant thinks he may feel lonely if he does not drink any alcohol in this community. He spoke of his friend (D)'s situation as an example "D doesn't like alcohol and doesn't drink alcohol on any occasion. We can understand his situation because $D$ is our friend and we know him so well. But if D joins in a social party and doesn't drink, people may think he disrespects them. This situation may lead him to troubles such as no one wants to invite him to a party' (FG03-M).

Participants consider alcohol use as a normal behaviour because it is accepted by most of the Vietnamese population. They believe alcohol consumption is a way for medical students to improve relationships with their friends, teachers, and colleagues at the university and their future career.

\section{Medical students' binge drinking experience: 'If they drink homebrew rice wine, you should count by barrels'.}

Participants believe binge drinking happens infrequently among medical students. However, most participants engage in or witness binge drinking occasions among medical students. Some participants report having a low tolerance for alcohol and feel intoxicated after 2-3 drinks but others can drink more than 10 cans of beer on one occasion. The participants agree it is difficult to join in a party without drinking alcohol or just consuming 1 or two drinks and stopping because of an accepted informal drinking culture among Vietnamese people and 'drinking depends on regional culture, your knowledge, circumstances of your family, and your place where you live... or people around you'(IV06-M). The participants confirm medical students experience binge drinking when they drink with their friends, teachers, and others in a group or team. Friends and teachers are found as normative referent groups who approve of medical students' binge drinking. Most participants experience binge drinking at parties, celebrations, camping festivals, sport competitions, and when they are experiencing bad feelings. Some participants mention that medical students binge drink only when they are free the next day. These circumstances are control beliefs that enable medical students to engage in binge drinking occasions.

FG02-F "I think binge drinking happens infrequently. It depends on the types of party you were joining. If the party was really fun and we didn't have to study/work on the next day, we would drink until we couldn't drink more"

The types of alcohol medical students choose when binge drinking are beer or homebrew rice wine. Participants say medical students rarely buy wine in shops because they are afraid of its quality. They only drink wine when someone orders wine from their hometown. The participants believe medical students are rarely drunk and still in control of their behaviour after drinking even when participating in binge drinking occasions. They believe this because medical students mostly drink at homes, go to sleep if they are drunk, and their friends can help to prevent them from being out of control.

Overall, participants experience binge drinking at parties, celebrations if they have free time next day. They choose homemade wine rather than commercial products. Medical students consume alcohol at home where they feel safer than external environments if they are intoxicated.

\section{Medical students' binge drinking is influenced by peer pressure and group affiliation: 'If you refuse to drink, you may be excluded from the groups/teams'.}

Participants mention medical students do not drink alcohol without a reason. The most frequent reason for binge drinking is to have fun or to share something with others. These reasons are examples of TPB behavioural beliefs which are the perceived advantages of binge drinking among medical students. Some medical students start to drink alcohol when they begin studying at the university and have become binge drinkers because they are influenced by the older students' drinking behaviour. Older students are considered as another normative referent group who approve of medical students binge drinking.

FG03-M "I often drank under peer pressure. ... I was influenced partly by the older students. I'm a class leader, so to keep the relationships in the university, I often had to drink with the students at higher levels. This is one reason that allows me to drink".

Participants think female students can easily decline to drink because females are considered as the weaker sex and 'have the right to decline' (FG01-M). Male students cannot refuse a drink when joining in drinking sessions. If male students say 'no' when invited to drink, it may be considered as lack of respect to other people who tried and they may be 'excluded from the groups/teams' (FG02-F). This control belief enables male students to engage in binge drinking occasions. Male students experience more peer pressure than females in drinking occasions due to the traditional opinion that 'A man without alcohol is 
like a flag without wind' ('Nam vô tửu như kỳ vô phong') and drinking alcohol to 'prove their manhood' (FG02-F). In female students' views, male students themselves create pressure to drink more. Male students mention a variety of reasons to ask others to have a drink.

FG02-F "Males have numerous reasons to ask others to drink. For example, if someone arrives late to a party, he would have to drink instead of apologising and others in the party would be witness to his apology by drinking with him"

Drinking is considered as a way of sharing among groups of friends. Sharing things that are hard to say is a perceived advantage (behavioural belief) of binge drinking behaviour among medical students. The close relationship between best friends and among medical students who are participating in groups/teams/clubs at the university influences drinking behaviour among medical students. Participants mention medical students who come from the Central Region of Viet Nam drink more alcohol and more frequently than others. These students have a close relationship with others from their region. They hold many meetings to share the difficulties of life and improve their friendship. In these meetings, alcohol is often consumed.

Participants who join in groups/teams/clubs such as the Youth group, Voluntary student team, and Sport clubs believe they have more opportunities to participate in binge drinking occasions than other medical students and became a 'strong drinker'. In particular, participants discuss that being leaders of these groups/teams/clubs means you have to drink more alcohol because a leader has responsibility to keep and improve relationships with teachers and other groups/teams/clubs through drinking more alcohol during drinking occasions to show his/her respect to others.

FG04-F "Students in groups/teams drink more frequently. I also joined in a voluntary group in the past. We joined in many activities... These activities were also our drinking occasions. We even sometimes drank overnight".

Peer pressure is an important factor leading to binge drinking behaviour among male medical students and serves as a control belief that enables medical students to binge drink. Male medical students have numerous reasons for succumbing to pressure to drink more alcohol. Medical students' alcohol consumption is influenced by others, especially the more mature students in drinking occasions. Group affiliation creates more pressure for medical students to drink alcohol.

\section{DISCUSSION}

This study showed in-depth insights into alcohol drinking behaviours among medical students. Participants believe medical students are not binge drinkers and drink less alcohol than other students. An international study conducted in 17 countries across Asia, Africa, and the Americas which compared the health risk behaviour between health science and non-health science students found health science students (including medicine, medical services, nursing, and dental services) engaged in significantly fewer binge drinking occasions than non-health science students $(9.1 \%$ compared with $16.9 \%) .^{31}$ In Viet Nam, some studies investigated the alcohol drinking proportion among medical students and other students separately. ${ }^{32}{ }^{33}$ It is difficult to confirm if medical students drink less than other students because these studies used different measures of alcohol consumption and at different points of time. More research is needed to confirm if a difference exists.

Vietnamese medical students in this study do not know the definition of binge drinking but feel confident about their knowledge in relation to alcohol use, and believe medical students do not have a problem with drinking and are not binge drinkers. However, they report many binge drinking occasions in which they participate or witness among medical students. This contrariety raises a question about medical students' beliefs about binge drinking. Some international studies found students believe they drink less than the majority of their peers, yet they consume more. ${ }^{34}{ }^{35}$ More studies need to be conducted to understand this complexity of understanding among Vietnamese medical students.

Participants believe drinking alcohol is normal and a part of the culture in Viet Nam. This attitude was found to affect alcohol consumption and intoxication of Vietnamese medical students. ${ }^{15}$ The same situation was found in Ireland and Denmark. ${ }^{36}{ }^{37}$ The culture of drinking is reproducing itself with new generations adapting and incorporating existing habits. This perspective is reflected in this present study where medical students are socialised to adapt to the drinking culture among friends and influenced by the older students. Future studies should examine factors relating to sociocultural factors, especially influences due to regional culture which affect drinking behaviour among medical students.

In the view of participants, alcohol consumption occurs among medical students to enhance other activities rather than drinking itself being the focus. Medical students in this present study consider drinking as a way to create and improve relationships with friends, teachers, and other people and to express their respect to others in drinking occasions, and that becoming a 'strong drinker' may be helpful for their future careers. This perception needs to be re-examined broadly among Vietnamese medical students and physicians, especially as previous research shows that the drinking behaviour of medical students and physicians may influence their attitudes and practices in relation to alcohol counselling for patients and the more frequently they drink alcohol, the less likely they counsel their patients about alcohol. ${ }^{12} 14$ However, this view among the participants aligns with the socially accepted view in Viet Nam that drinking will enhance social and business relationships, and maintain 
careers. ${ }^{38}$ A study found the main motive of Vietnamese students in Ho Chi Minh city to drink alcohol was to create a favourable introduction to their friendships and working relationships. ${ }^{39}$ In this sense, alcohol becomes a mean to create social bonds and friendships. ${ }^{37}$ Saying no to alcohol in a drinking occasion may be similar to rejecting fellow students or colleagues.

Group affiliation and value judgements of friends are important factors related to binge drinking among medical students in this study. These medical students indicate they engage in binge drinking occasions with friends, particularly in large groups/teams/clubs, and this participation creates pressure to drink more. A study found most Vietnamese students including medical students often drank with their friends and nearly half of them drank in large groups of 4-9 friends. ${ }^{17}$ Group affiliation and value judgements of friends is related to binge drinking among university students from 50 countries across Europe, North America, and the Asia-Pacific region. ${ }^{40}$ A study found students who have more social relationships with binge drinkers are more likely to binge drink and that being at the core of a group of friends put individuals at greater risk of binge drinking. ${ }^{41}$

In this present study, peer pressure is a factor underlying binge drinking behaviour among male participants and males drink more alcohol than females. These male medical students experience peer pressure from older medical students and their close friends. Many studies have confirmed this finding among Vietnamese students ${ }^{1517}$ as well as Vietnamese people in general. ${ }^{42}$ In Viet Nam, male students are influenced by their peers and others to display behaviours seen as 'manly'. ${ }^{43}$ In previous research, it has been showed that drinking pressure was created more by experienced medical students towards their younger 'fresher' peers rather than from peers in the same year, ${ }^{6}$ and higher for students with reciprocated ties than for those with non-reciprocated ties. ${ }^{41}$ Future studies and drinking interventions should consider factors of peer pressure and group affiliation, and focus more on male students' drinking behaviour.

The present study has identified salient TPB beliefs underlying binge drinking behaviour among a group of Vietnamese medical students. The behavioural beliefs reflecting the advantages of binge drinking among medical students include having fun, socialising with others, improving relationships, and sharing things that are hard to say. Friends, teachers, and older students serve as normative referent groups who approve of medical students engaging in binge drinking occasions. Participants experience circumstances that enable them to binge drink such as celebrations, peer pressure, to forget bad things, and showing their drinking ability or respect to the people they are drinking with, in particular older people; while societal expectations prevent them from, and serve as barriers to binge drinking. These findings are consistent with previous research by French and Cooke. ${ }^{26}$ Future research and interventions applying the TPB framework should consider these important underlying beliefs to understand and address binge drinking behaviour among medical students.

\section{CONCLUSION}

This study provides insights into the factors underlying drinking behaviour among a convenience sample of Vietnamese medical students and reveals a dichotomy between the belief of not being binge drinkers yet experiencing many binge drinking occasions. The findings of the present study can help to inform future research directions. It appears that the TPB may serve as a useful framework for a predictive study examining the influences on binge drinking among a larger sample of Vietnamese medical students. Based on the themes identified in the present study, the addition of other variables such as group norms, role identity, and drinking culture in the prediction of binge drinking among Vietnamese medical students may be beneficial. If future research identifies the predictors of binge drinking intention and behaviour among Vietnamese medical students, these findings can be used to inform stronger evidence-based approaches to prevent binge drinking. Efforts to understand and reduce risky drinking behaviours among medical students is essential as the providers of future alcohol use related consultations for the population.

Acknowledgements The authors thank staff of the Centre for Educational Testing and Quality Assurance at the Medical University in Viet Nam for support to the implementation of this study.

Contributors TTHN designed the study, conducted the focus groups and interviews, analysed and interpreted data and wrote the manuscript. MCS provided guidance on the recruitment, conducting focus groups and interview, analysis, interpretation and reporting of findings, and revised drafts of the manuscript. KMW provided advice regarding the design, framework, and recruitment of the study, oversaw the development of fieldwork materials, reporting of findings, and revised drafts of the manuscript. RMY provided advice in relation to organizing and conducting the study, and feedback on manuscript drafts. All authors contributed to and approved the current version of the manuscript.

Funding The authors have not received any funding or benefits from industry or elsewhere to conduct this study.

Competing interests None declared.

Patient consent Not required.

Ethics approval This study received ethics approval from Queensland University of Technology, Australia (1600000785) and the Medical University in Viet Nam (IRB00003121).

Provenance and peer review Not commissioned; externally peer reviewed.

Data sharing statement To access supplementary appendix 1 , contact the corresponding author at tt26.nguyen@hdr.qut.edu.au. Supplementary appendices 2 and 3 are available to access online.

Open Access This is an Open Access article distributed in accordance with the Creative Commons Attribution Non Commercial (CC BY-NC 4.0) license, which permits others to distribute, remix, adapt, build upon this work non-commercially, and license their derivative works on different terms, provided the original work is properly cited and the use is non-commercial. See: http://creativecommons.org/licenses/by-nc/4.0/

(c) Article author(s) (or their employer(s) unless otherwise stated in the text of the article) 2018. All rights reserved. No commercial use is permitted unless otherwise expressly granted.

\section{REFERENCES}

1. World Health Organization. Global status report on alcohol and health 2014. Geneva: World Health Organization, 2014. 
2. Jennison KM. The short-term effects and unintended long-term consequences of binge drinking in college: a 10-year follow-up study. Am J Drug Alcohol Abuse 2004;30:659-84.

3. Tavolacci MP, Boerg E, Richard L, et al. Prevalence of binge drinking and associated behaviours among 3286 college students in France. BMC Public Health 2016;16:178.

4. Yi S, Ngin C, Peltzer K, et al. Health and behavioral factors associated with binge drinking among university students in nine ASEAN countries. Subst Abuse Treat Prev Policy 2017;12:32.

5. Trostler M, Li Y, Plankey MW. Prevalence of binge drinking and associated co-factors among medical students in a U.S. Jesuit University. Am J Drug Alcohol Abuse 2014;40:336-41.

6. Black LF, Monrouxe LV. 'Being sick a lot, often on each other': students' alcohol-related provocation. Med Educ 2014;48:268-79.

7. Arnett JJ. The developmental context of substance use in emerging adulthood. J Drug Issues 2005;35:235-54.

8. Melaku L, Mossie A, Negash A. Stress among medical students and its association with substance use and academic performance. $J$ Biomed Educ 2015;2015:1-9.

9. Newbury-Birch D, Lowry RJ, Kamali F. The changing patterns of drinking, illicit drug use, stress, anxiety and depression in dental students in a UK dental school: a longitudinal study. Br Dent J 2002;192:646-9.

10. Bakhshi S, While AE. Health professionals' alcohol-related professional practices and the relationship between their personal alcohol attitudes and behavior and professional practices: a systematic review. Int J Environ Res Public Health 2013;11:218-48.

11. Duperly J, Lobelo F, Segura C, et al. The association between Colombian medical students' healthy personal habits and a positive attitude toward preventive counseling: cross-sectional analyses. BMC Public Health 2009;9:218-18.

12. Frank E, Segura C, Shen $\mathrm{H}$, et al. Predictors of Canadian physicians' prevention counseling practices. Can J Public Health 2010;101:390-5.

13. Yu Y, Yang Y, Li Z, Zf L, et al. The association between medical students' lifestyles and their attitudes towards preventive counseling in different countries. BMC Public Health 2015;15:1124.

14. Frank E, Elon L, Naimi T, et al. Alcohol consumption and alcohol counselling behaviour among US medical students: cohort study. BMJ 2008;337:a2155-53.

15. Le TTA N, Nguyen TMN, Nguyen TTN, et al. Knowledge, attitude and behavior on drinking beer and alcohol among the medical students of Hai Phong University of Medicine and Pharmacy in 2015. Viet Nam Journal of Preventive Medicine 2015;171.

16. Pham DB, Clough AR, Nguyen HV, et al. Alcohol consumption and alcohol-related problems among Vietnamese medical students. Drug Alcohol Rev 2010;29:219-26.

17. Diep PB, Tan FE, Knibbe RA, et al. A multilevel study of students in vietnam: drinking motives and drinking context as predictors of alcohol consumption. Int J Environ Res Public Health 2016;13:710.

18. Diep PB, Schelleman-Offermans K, Kuntsche E, et al. Direct and indirect effects of alcohol expectancies through drinking motives on alcohol outcomes among students in Vietnam. Addict Behav 2016;52:115-22

19. Cuong DV, Hanh VTM. Assessment of situation of alcohol and beer abuse in Vietnam. Hanoi: Ministry of Health, 2007.

20. Ajzen I. From intentions to actions: a theory of planned behavior. In: Kuhl J, Beckmann J, eds. Action control: from cognition to behavior. Berlin, Heidelberg: Springer Berlin Heidelberg, 1985:11-39.

21. Ajzen I. The theory of planned behavior. Organ Behav Hum Decis Process 1991;50:179-211.
22. Glassman T, Braun RE, Dodd V, et al. Using the theory of planned behavior to explain the drinking motivations of social, high-risk, and extreme drinkers on game day. J Community Health 2010;35:172-81.

23. Ross A, Jackson M. Investigating the theory of planned behaviour's application to binge drinking among university students. J Subst Use 2013;18:184-95.

24. Armitage CJ, Arden MA. A volitional help sheet to reduce alcohol consumption in the general population: a field experiment. Prev Sci 2012;13:635-43.

25. Armitage CJ, Rowe R, Arden MA, et al. A brief psychological intervention that reduces adolescent alcohol consumption. J Consult Clin Psychol 2014;82:546-50.

26. French DP, Cooke R. Using the theory of planned behaviour to understand binge drinking: the importance of beliefs for developing interventions. Br J Health Psychol 2012;17:1-17.

27. Hagger MS, Lonsdale A, Chatzisarantis NL. A theory-based intervention to reduce alcohol drinking in excess of guideline limits among undergraduate students. Br J Health Psychol 2012;17:18-43.

28. Viet Nam Government. Decision No 244/QD-TTg on National Policy of Preventing Harms of Abusing Alcoholic Bevarages by 2020. Hanoi: The Prime Minister of Viet Nam, 2014.

29. Ajzen I. Constructing a theory of planned behaviour questionnaire, 2006.

30. Liamputtong P. Qualitative research methods. 4th ed. South Melbourne: Oxford University Press, 2013.

31. Peltzer K, Pengpid S, Yung TK, et al. Comparison of health risk behavior, awareness, and health benefit beliefs of health science and non-health science students: An international study. Nurs Health Sci 2016;18:180-7.

32. Diep PB, Knibbe RA, Giang KB, et al. Alcohol-related harm among university students in Hanoi, Vietnam. Glob Health Action 2013;6:59-68.

33. DuongTran P. Sociocultural perceptions and patterns of cigarette and alcohol use among college students in Vietnam. Asian Social Work and Policy Review 2008;2:149-58.

34. Crawford LA, Novak KB. Resisting peer pressure: characteristics associated with other-self discrepancies in college students' levels of alcohol consumption. Journal of Alcohol and Drug Education 2007;51:35-62.

35. Neighbors C, Lee CM, Lewis MA, et al. Are social norms the best predictor of outcomes among heavy-drinking college students? J Stud Alcohol Drugs 2007;68:556-65.

36. Hogan G, O'Loughlin D. A qualitative study of Irish postgraduate students' alcohol consumption. J Subst Use 2014;19:373-7.

37. Ladekjær Larsen E, Smorawski GA, Kragbak KL, et al. Students' drinking behavior and perceptions towards introducing alcohol policies on university campus in Denmark: a focus group study. Subst Abuse Treat Prev Policy 2016;11:17.

38. Lincoln M. Alcohol and drinking cultures in Vietnam: a review. Drug Alcohol Depend 2016;159:1-8.

39. TTL L. Drinking motives of university students in Ho Chi Minh City. Ho Chi Minh City: University of Education, 2013.

40. Russell-Bennett R, Hogan S, Perks K. A qualitative investigation of socio-cultural factors influencing binge-drinking: a multi-country study, 2010.

41. Lorant V, Nicaise P. Binge drinking at University: a social network study in Belgium. Health Promot Int 2015;30:675-83.

42. Giang KB, Van Minh $\mathrm{H}$, Allebeck P. Alcohol consumption and household expenditure on alcohol in a rural district in Vietnam. Glob Health Action 2013;6:18937-8.

43. Kaljee LM, Genberg BL, Minh TT, et al. Alcohol use and HIV risk behaviors among rural adolescents in Khanh Hoa Province Viet Nam. Health Educ Res 2005;20:71-80. 